Marie VOŽDOVÁ

Université Palacký, Olomouc

\title{
LA DÉMYTHISATION D'UN MYTHE LITTÉRAIRE : ROBESPIERRE ET PAUVRE BITOS OU LE DÎNER DE TÊTES
}

Le personnage historique contradictoire de Maximilien de Robespierre apparaît rarement dans les œuvres littéraires et y est interprété de façons différentes. ${ }^{32}$ Dans son Dictionnaire des mythes littéraires, Pierre Brunel présente Robespierre comme un héros mythique dont la légende a une double face. Il incarne d'un côté le mythe de l'apôtre de la révolution sociale et du martyr de thermidor, et de l'autre symbolise le monstre inhumain qui se nourrit de la chair humaine. ${ }^{33}$ Dans notre article, nous allons étudier les pièces de deux dramaturges du théâtre français qui, à travers la même histoire de Robespierre, expriment des idées très différentes. Pour Romain Rolland, auteur aux positions marxistes, Robespierre constitue le vrai mythe du révolutionnaire idéal, incorruptible et voué au peuple. Son personnage joue un rôle important dans le cycle dramatique rollandien du Théâtre de la Révolution. ${ }^{34}$ Jean Anouilh, auteur aux positions idéologiques opposées, et ennemi de toutes les formes de cruauté ainsi que de l'assassinat légal, décompose ce mythe rollandien et en soumettant le personnage de Robespierre à son regard critique, montre la double face de son mythe.

Romain Rolland était fasciné par la thématique de la Révolution française, par les passions qui poussaient les masses et par les génies héroïques de l'action. Obsédé par la mort héroïque, il a voulu, dans l'image de son Robespierre, exalter l'héroïsme et l'idéalisme. Avant d'être le héros principal de la pièce intitulée de son nom, le personnage de Robespierre apparaît dans les pièces Le 14 juillet $^{35}$ et Danton $^{36}$. L'image du héros évolue dans la mesure où son rôle dans l'histoire devient plus important. Dans la présentation des personnages située chaque fois avant la pièce, nous pouvons lire : " Maximilien de Robespierre, 31 ans, député à la Constituante... sa figure est plus pleine, plus molle, elle n'a pas encore été [...] creusée par la fatigue et la responsabilité. Une flamme blanche. L'âme n'a pas pris conscience de sa force intérieure, mais cette force est là, muette, se manifestant seulement par l'absolu renoncement qu'on sent qu'il a déjà fait de sa vie, sans croire au succès, par un stoïcisme hautain, pessimiste et glacé » (ROLLAND, 1926 : 6) et puis : «Robespierre, 36 ans. Taille moyenne, complexion délicate. Cheveux

\footnotetext{
${ }^{32}$ Cf. La Mort de Danton de Georges Büchner de 1835 où Danton et Robespierre incarnent les héros mythiques.

${ }^{33}$ Cf. BRUnel Pierre (1988), Dictionnaire des mythes littéraires, Monaco, Editions du Rocher, p. $1056,1216-1222$.

${ }^{34}$ Cf. Rolland Romain (1926), Théâtre de la Révolution. Le 14 Juillet. Danton. Les Loups, Paris, Librairie Ollendorff. Cette édition contient aussi Préface au Théâtre de la Révolution écrite par Romain Rolland en janvier 1909.

${ }^{35}$ La pièce de théâtre Le 14 juillet a été écrite par Romain Rolland en 1902 et elle a été publiée avec les autres œuvres du Théâtre de la Révolution en 1926.

${ }^{36}$ La pièce de théâtre Danton a été écrite par Romain Rolland en 1900 et elle a été publiée avec les autres œuvres du Théâtre de la Révolution en 1926.
} 
châtains. Yeux vert-sombre, grands, fixes, et myopes. Grosses besicles, relevées sur le front. Nez droit, légèrement retroussé du bout. Teint pâle. Lèvres fines, à 1'expression dédaigneuse, inquiétante, non sans attrait. » (ROLLAND, 1926 : 156).

Comme il est connu que la pièce Danton a été en réalité écrite par Romain Rolland deux ans avant Le 14 juillet, l'intention de l'auteur de souligner chaque fois certains traits de caractère de son héros et de donner ainsi plusieurs portraits partiels qui seulement assemblés traduiront le personnage historique en entier, semble évidente. Rolland, en auteur omniprésent, prévoit ce qui se cache dans le visage de son héros, ressent sa force intérieure, son ardeur potentielle pour l'idée de la Révolution accompagnée par un certain manque de faiblesse humaine et de sentiment.

Dans Le 14 juillet Robespierre n'apparaît que quatre fois en tant que personnage secondaire qui accompagne les chefs révolutionnaires connus par le peuple. Il est introduit comme le député d'Arras, inconnu dans le milieu des chefs révolutionnaires, protégé par Camille Desmoulins et présenté par lui au peuple de Paris. Grâce à ses amis, Robespierre essaie pour la première fois de lire son discours sur la Déclaration des Droits au peuple, mais, à cause de sa voix faible et tremblante, personne ne l'écoute. Ne parlant que de la cause du peuple, il se montre ennemi de l'amour et de toute relation amoureuse qui affaiblit les gens et les emmène loin de l'action révolutionnaire. Il reproche à Camille Desmoulins son amour pour Lucile: «Tu oublies ton devoir. [...] Il ne s'agit plus d'amour aujourd'hui [...] Le nom de l'amour est banal, sa réalité est rare. » (RoLLAND, 1926 : 99, 100, 185). Romain Rolland, tout en conservant toujours la compassion de l'auteur pour son personnage, montre ses points faibles concernant sa relation aux femmes et à l'amour : « [...] quand on dit devant lui une gauloiserie ? Son front se plisse de grandes rides [...] il crispe les mains, il grimace, comme un singe qui a mal aux dents. [...] C'est de Rousseau qu'il tient sa haine de Voltaire. » (RollaND, 1926: 175). Néanmoins, déjà dès la première sortie de Robespierre sur la scène et d'après la description faite par Rolland, on sent son idéalisation de la part de l'auteur, le personnage incarne la vision du révolutionnaire idéal, doué pour la cause du peuple. (Il est évident qu'il se fait très difficile d'obéir aux exigences de Rolland dramaturge et de faire revivre ce personnage sur la scène théâtrale.) $)^{37}$

Robespierre dans la pièce Danton, c'est déjà un homme au pouvoir qui fait peur à ses anciens amis et qui décide au jour le jour de leur condamnation à mort tout en croyant à la nécessité de la vertu révolutionnaire. Ainsi, poussé par SaintJust, il laisse guillotiner aussi Danton et même, son ami Camille Desmoulins. Robespierre est comme obsédé par la mort, il ne fait confiance à personne et voit partout des traitres et des ennemis. «Je me défie de tous les hommes. Je lis le mensonge dans les regards, je vois la ruse embusquée sous les protestations. Leurs yeux, leur bouche, leurs serrements de main, leur corps tout entier ment. Le soupçon empoisonne toutes mes pensées. J'étais fait pour des sentiments plus doux. J'aime les hommes, je voudrais croire en eux. Mais comment y croire

\footnotetext{
${ }^{37}$ Jusqu'aujourd'hui, à notre connaissance, Robespierre n'a été joué que deux fois. Une première fois en 1952 à Leipzig en Allemagne et une seconde fois en 1960 par le Théâtre d'Etat de Brno en Tchécoslovaquie.
} 
encore $[\ldots] \gg$ (RolLAND, 1926 : 206). Romain Rolland présente son Robespierre comme un personnage qui souffre d'être obligé de faire le mal pour la victoire de la cause révolutionnaire, ce qui est une idée admise par son inclination marxiste.

Par la pièce de théâtre intitulée Robespierre, Romain Rolland en 1939 à l'âge de soixante-douze ans atteint le sommet de son épopée dramatique consacrée à la Révolution. D'après l'auteur, ce drame représente «la tragédie des hommes qui ont choisi, contre leur cœur, d'être des instruments implacables du Destin, d'accomplir dans le sang les actes nécessaires à la libération et à l'essor de la nouvelle humanité. Ils savent qu'au regard de la conscience individuelle - la leur ces actes sont souvent des crimes. Et ils en sont quelquefois accablés. Mais ils savent que, sans ces crimes, la Révolution serait arrêtée et ils acceptent pour la servir, le rôle terrible de la hache $»^{38}$ Au niveau du sujet, l'œuvre est liée directement à la pièce Danton. Rolland y peint la période entre la mort de Danton et la chute de Robespierre. Danton et Desmoulins se trouvent sur la charrette qui les emmène vers la guillotine. Les mots criés par Danton « J'ouvre la fosse, tu m'y suivras... à bientôt ...! » (ROLLAND, 1939:13) du commencement de la pièce suggèrent le destin de Robespierre et font sentir sa fin. Dans le personnage de Robespierre l'auteur essaie de montrer, conformément à son idée de pluralité des âmes dans chaque homme, le révolutionnaire dur et aussi l'homme sensible qui croit en Dieu, et souffre de causer de la douleur aux gens en voulant faire leur bien. Cette intention de l'auteur d'aller plus loin dans la peinture des qualités de caractère de son héros en comparaison avec les pièces précédentes traduit de nouveau sa foi idéaliste en la Révolution et en l'idée du peuple suprême.

Le motif du peuple pour lequel Robespierre se sacrifie se trouve, dans l'œuvre de Rolland, omniprésent. Déjà dans Danton, le personnage de Robespierre en tant que porte-parole de l'auteur, déclamait : «Dans le peuple est notre lumière, son salut est notre loi. » (ROLLAND, 1926 : 227).

Rolland, étant fasciné par le génie et la force de caractère de son héros, utilise une langue exaltée et lui fait prononcer des tirades entières en s'adressant au peuple et à la patrie : «Peuple... mon peuple ! ... Je suis à toi, je t'appartiens, rien n'est en moi qui ne soit tien. Prends-moi, bois-moi, reçois le sacrifice de tout mon être !... Peuple Sublime ! Heureux qui est né au milieu de toi ! Plus heureux qui peut mourir pour ton bonheur ! [...] O, ma patrie, terre délicieuse que la nature caresse avec tendresse. Tu es le siège de la gloire et de la liberté ! (ROLLAND, 1939 : 123, 124). Ces extases verbales déclamatoires durant lesquelles l'auteur s'oublie, contrastent avec le reste des paroles de Robespierre, avec sa dureté envers les individus et montrent son personnage fort invraisemblable et trop stylisé. Obsédé par la raison, Robespierre cherche toujours des ennemis autour de la Révolution « Nous ne sommes rien, et elle est tout. » (ROLLAND, 1939 : 129), la tentative de le tuer échoue et est suivie de nouvelles condamnations à mort. Il se sent fort, toujours s'appuyant sur le peuple: «Robespierre : Je vais au peuple. L'unique ami. Mon maître [...]. - Billaud: Nous te chassons du Comité ! Robespierre : Et moi, je vous chasse de la République... » (RolLAND, 1939 : 143). L'auteur ajoute une image amère de la trahison du peuple infidèle «Voix de

\footnotetext{
${ }^{38}$ Noté par Romain Rolland dans son Journal en octobre 1931. Cité d'après DUCHATELET Bernard (2002), Romain Rolland tel qu'en lui-même, Paris, Albin Michel, p. 303.
} 
Robespierre : O peuple, mon peuple, mon unique ami ! Quoiqu'il arrive, restons ensemble ! [...] - La voix du peuple : Je ne te quitte point, je te suivrai jusqu'où tu vas. - Voix de Robespierre : Et sais-tu où ? - La voix du peuple : A la guillotine ! ... Tu dors, traître... ! Réveille-toi ! » (RoLland, 1939 : 304, 305).

Dans la philosophie de Romain Rolland, Maximilien Robespierre est quelqu'un qui se sacrifie pour le bonheur des autres. D'ici plusieurs allusions utilisées par l'auteur à la vie de Jésus et à son sacrifice pour l'humanité. Déjà dans Danton, Robespierre dit un peu mystérieusement aux gens qui l'adorent : « [...] et pourtant je vous quitterai plus tôt que vous ne pensez. » (RoLLAND, 1926 : 195). C'est d'ailleurs dans la même pièce que Jésus est appelé par les révolutionnaires «le sans-culotte Jésus » (RoLlAND, 1926: 238). Les cris de la foule extasiée désignent Robespierre pendant son discours comme un saint et même comme Jésus. Les scènes 12-17 de l'acte II où Robespierre déjà blessé et arrêté attend la mort, se passent pendant une nuit jusqu'au matin. La foule désigne ses disciples, mais ils le renient et crachent sur lui. Rolland y introduit aussi un passage lyrique contrastant avec l'atmosphère du reste du livre, et où Robespierre récapitule avant sa mort dans ses souvenirs toute sa vie, son enfance à Arras, ses années adolescentes au collège où il était méprisé pour sa pauvreté, l'absence de la mère et son inclination pour la lecture, son arrivée à Paris, l'enthousiame de la foule et son triomphe et aussi la haine de ce même peuple, et anticipe sa mort à la guillotine qui l'attend.

A une vingtaine d'années de Romain Rolland, c'est Jean Anouilh qui consacre entre autres au personnage de Robespierre son drame Pauvre Bitos ou Le Dîner de têtes. L'œuvre est publiée en 1956 et 1958 et fait partie des pièces grinçantes. ${ }^{39}$ Dans la pièce d'Anouilh, deux réalités se superposent, l'une lointaine du dixhuitième siècle et l'autre plus récente concernant l'époque de l'épuration après la Libération. A la différence de Romain Rolland, Anouilh est contre tout contenu idéologique de l'œuvre. En posant son regard ironique sur la société de l'aprèsguerre ainsi que sur celle de la Révolution française, il trouve dans les deux époques des parallèles dans le comportement des gens.

La situation initiale de la pièce présente un riche bourgeois, Maxime, qui reçoit en héritage l'ancien prieuré de Carmes où, en 1792, les Jacobins faisaient leurs réunions et en 1793 y était installé le Tribunal révolutionnaire. Avant de le vendre, il veut y organiser un dîner de têtes ayant pour but la punition d'André Bitos, ancien compagnon de collège et fils d'une pauvre blanchisseuse devenu procureur, qui participe avec ferveur aux procès de l'épuration. Parmi les invités il y a des jeunes notables de province dont chacun doit jouer le rôle d'un personnage vivant pendant la Révolution française. Ils doivent tous étudier le chapitre sur la Terreur dans le Manuel d'histoire Malet-Isaac et savoir animer pendant le repas le débat sous leurs masques autorisés, dans le but d'enivrer Bitos et de le faire parler. Anouilh fait jouer à ses héros les rôles des célèbres révolutionnaires, comme Danton, Tallien, Mirabeau ou Camille Desmoulins qui ont entouré Robespierre. Le rôle de Robespierre est attribué à André Bitos.

\footnotetext{
${ }^{39}$ Le drame Pauvre Bitos ou Le Dîner de têtes a été publié d'abord séparément en 1956 dans les Editions de la Table ronde, et puis, en 1958, il faisait partie de l'ensemble des pièces grinçantes. Cf. ANouilh Jean (1958), Pauvre Bitos ou Le Dîner de têtes, Pièces grinçantes, Paris, La Table ronde.
} 
Anouilh développe un parallèle entre Robespierre qui fait tuer des gens pour la vertu de la Révolution et André Bitos qui dans le cadre de son travail, avec le même enthousiasme aveugle, condamne des gens à la mort pour leur collaboration avec les nazis. L'effet d'une double réalité est renforcé par sa triple réalisation : tout d'abord le décor possède une double authenticité, les murs de l'ancien couvent sont des témoins de l'histoire révolutionnaire mais servent aussi de cadre au sujet actuel. De même, les protagonistes à part leurs têtes de l'époque portent, à l'exception de Bitos qui s'est trompé de coutume et est habillé entièrement comme Robespierre, des habits contemporains. Le double rôle de Bitos-Robespierre permet à l'auteur d'identifier les deux personnes et c'est sur cette identification qu'est construite toute la pièce. Bitos, l'ancien boursier pauvre, toujours premier mais victime des mauvaises plaisanteries des fils de bonnes familles bourgeoises, s'approche dans son désir de pureté morale, de son personnage double : «Il se croit Robespierre. La justice immanente est en marche et c'est lui. » (ANOUILH, 1958 : 377). Anouilh mélange les moments de la vie de son personnage Bitos à ceux du personnage joué par lui, c'est-à-dire de Robespierre. Ainsi, il crée un sujet plus compliqué et plus complexe, l'utilisation du procédé de la mise en abîme lui permet de montrer la psychologie de son héros de différents points de vue. Dans les pièces à venir, le «théâtre dans le théâtre » deviendra sa formule récurrente. ${ }^{40}$ Les deux niveaux d'histoires s'entremêlent, le premier commence par le jeu de rôles, puis le jeu s'efface jusqu'à l'identification absolue des deux personnages pour, à la fin de la pièce, revenir de nouveau vers ce jeu de rôles. Il s'agit de la première pièce dans laquelle Anouilh joue sur le glissement entre différentes strates chronologiques et utilise à l'aide du changement de l'éclairage le jeu de l'ombre.

Le portrait physique du personnage de Robespierre peint par Anouilh montre un homme " raide, maladroit, les mains aux doigts serrés au bout de bras laides » (ANOUILH, 1958: 403), qui dans sa marche se heurte aux portes, renverse les chaises et ne sait que marcher droit. L'auteur le présente comme un automate qui n'a jamais ni souri ni embrassé personne et dont les mains n'ont jamais travaillé. Il ne sait rien créer à partir d'elles, n'étant capable que de parler et de détruire la vie. Plusieurs fois, en liaison avec Bitos-Robespierre, Anouilh utilise le mot « vertu», mais qui, dans son cas est interprété par l'auteur presque comme son contraire, un vice dangereux pour les autres : «La rigueur et la vertu du peuple sont dans nos murs » (ANOUILH, $1958: 377$ ), dit Maxime en parlant de la présence de Bitos dans la ville. Avec une ironie amère, Anouilh parle de l'un des jeunes aristocrates, qui a «repris de nombreuses usines de papa», mais Bitos dont la mère était blanchisseuse, «n'a pas daigné continuer le fonds de blanchisserie de sa mère. Il s'est fait substitut, pour faire la lessive en plus grand. » (ANOUILH, 1958: 384). De même Robespierre comme le double familier de Bitos est obsédé par la pureté et la vertu, déteste la saleté et nettoie sans cesse ses habits. Avec ce nettoyage physique va de pair son nettoyage révolutionnaire, la suppression des coupables. Quand à Bitos, lui, dans son fanatisme de pureté absolue, a aussi cherché des coupables. Il a fait condamner à mort «dans un sursaut de vertu» un ami

\footnotetext{
${ }^{40}$ Cf. Malachy Thérèse (1978), Jean Anouilh, les Problèmes de l'existence dans un théâtre de marionnettes, Paris, Nizet. Voir notamment le chapitre Théâtre dans le théâtre, p. 93-107.
} 
d'enfance. (ANOUILH, $1958: 385$ ). Comme Robespierre qui avait trahi et tué son ami Camille Desmoulins ainsi que détruit sa famille, Bitos condamne aussi et exécute son ami en ignorant la souffrance de la femme du condamné. Après l'exécution il choque son entourage parce qu'il regarde sa montre en disant: « Nous avons respecté l'horaire. » (ANOUILH, 1958 : 386) Le soir même, il achète une poupée très chère et l'envoie à la fille orpheline. Anouilh ajoute ironiquement la critique des valeurs et de l'hypocrisie de la société : «Une poupée qui fermait les yeux, disait papa et maman et faisait pipi. C'était d'ailleurs une poupée allemande car, si on exécutait encore, le commerce, lui, avait repris. » (ANOUILH, $1958: 386$ ).

Dans le double personnage de Bitos-Robespierre, l'auteur développe le motif du faux sauveur qui dans sa pensée détournée «se sacrifie» pour le bien de la nation. Ainsi Bitos ivre explique ses intentions de vouloir nettoyer la France après la guerre, de la faire propre et de choisir certaines personnes comme victimes de l'épuration pour que les autres se sentent lavés et plus innocents. Hypocrite, il prétend souffrir en même temps que les condamnés et se torturer, de même que son double Robespierre qui dans son fanatisme démagogue peut tuer sans scrupules ses amis et jouer à la fois au martyr : "Chaque goutte de sang qu'il me faut verser, je la tire de mes propres veines : sachez-le, mais il n'est pas d'ami que je ne sacrifierai à mon devoir, sachez-le aussi. » (ANOUILH, 1958 : 462). Au nom du même devoir, Bitos exécute son ami Lucien pour la collaboration. A ce motif de sauveur hypocrite Anouilh ajoute celui de la mère qui renie son fils. Bitos s'était toujours vanté de l'effort de sa mère et de ses mains rouges de blanchisseuse grâce auxquelles il était devenu ce qu'il était. Mais ce n'était que paroles. Quand il a demandé la mort de Lucien, cette mère est venue avec celle de Lucien, toutes les deux l'ont supplié à genoux, pleurant et lui embrassant les jambes. Le fils a, dans son fanatisme et sa haine omniprésente, regardé sa mère s'humilier devant lui et préféré se laisser gifler par la femme malheureuse que retirer la punition contre son ami.

Anouilh développe les mauvaises qualités de caractère de son héros, sa haine contre tout le monde, son orgueil qui prend sa source dans les années de l'enfant humilié, méprisé et mal aimé. Il est ivre de pouvoir et du désir de revanche. Sa pauvreté vécue explique toutes ses violences et toute la haine de la race humaine et du bonheur des autres. Aveuglé par son envie de vengeance, comme un enfant maladroit qui n'a pas su grandir, il est contre tous, contre les anciens compagnons qui sont devenus de vrais hommes, contre leurs amours et leur vie familiale quotidienne : «Je hais encore les hommes... [...] Ça leur apprendra à me faire peur... [...] mes belles, vos gros amants, vos taureaux bien membrés, vos mâles, vous ne savez pas qu'il suffit qu'il paraisse et qu'il les regarde, le petit Robespierre qui est si maigre et si laid [...] Putains! Je vous apprendrai, moi ! Je vous ferai veuves. Il n'y aura plus personne dans vos lits ! [...] Beaux Français, beaux messieurs, beaux mâles, je vous ferai passer le goût de vivre et d'être des hommes! Je vous ferai propres, moi !». (ANOUILH, $1958: 466-468$ ). La bouche toujours pleine du peuple et de son bonheur, en réalité il se révèle être un hypocrite qui hait et méprise ce même peuple dont il est issu : «Je n'ai pas d'ami. La poigne. C'est ma seule amie. Faire ce qui se doit durement, contre tous. Mais je n'aime personne. Même pas le peuple. Il pue. [...] Et j'ai horreur de ce qui pue 
[...] Le sentiment de vous faire peur, à tous, est doux aussi...» (ANOUILH, 1958 : 494-495). Anouilh comprend ainsi le personnage de Bitos, et celui de Robespierre, comme un être faible qui dans son incorruptibilité et sa cruauté cachait en réalité sa peur devant le peuple, les femmes, l'amour physique (d'où sa vertu) et devant la vie elle-même. Habitué aux mauvais coups du destin et capable de leur faire face par sa haine, il ne sait même pas accepter l'aide et la compassion offertes. A la jeune fille Victoire qui seule prend pitié de lui et ne le ridiculise pas, il promet sa plus grande vengeance car cette bonté à elle le blesse et l'humilie encore plus que la haine.

L'auteur défait complètement ce mythe du révolutionnaire Robespierre, ne faisant de lui ni un sauveur ni un tyran, mais tout simplement un homme médiocre. Lui-même voit la force de la France chez les médiocres et se croit l'un d'entre eux. Dans le dialogue de son personnage avec Mirabeau il prend le parti des médiocres et menace par leur force qu'on peut exploiter. «Mirabeau: Je n'aime pas les médiocres. Robespierre: [...] millions de médiocres [...] sont la France. Mirabeau : Ils l'habitent ! Mais ce n'est pas eux qui l'ont faite ! [...] Les hommes qui ont fait la France n'avaient de commun avec eux que d'avoir deux bras et deux jambes, comme eux, mais [...] une tête qui les dépassait. Robespierre : Dans les années qui vont venir, nous allons devoir nous employer à les couper, ces têtes qui dépassent. » (ANOUILH, 1958: 446). Anouilh utilise intentionnellement le mot médiocres au lieu de pauvres ou peuple, car pour lui ceux qui commettent les crimes et se prêtent à la violence ne méritent pas d'être appelés par le grand nom de peuple. Si Bitos réclame la modestie de Robespierre envers le côté matériel de la vie (qui le 9 thermidor n'avait que des livres en sa possession et devait même le loyer à la famille Duplay) on constate par la bouche de Maxime qu' « au milieu des pires catastrophes, les Français n'ont jamais cessé de penser à bien manger.» (ANOUILH, $1958: 398$ ).

Anouilh déshonore dès le commencement de la pièce l'idée de la mémoire révolutionnaire auprès des générations contemporaines. Maxime veut vendre sa propriété à la Maison Shell. Le garage construit à la place où la société locale des Jacobins tenait ses réunions symbolise le mépris des jeunes aristocrates pour leurs ancêtres : «[...] cela apprendra à mes ancêtres à s'être laissé guillotiner comme des moutons [...] S'ils s'étaient tous barricadés ici et qu'ils y soient morts en se défendant comme des hommes, j'aurais conservé le bâtiment [...] je suis sentimental. » (ANOUILH, $1958: 375,376$ ). Le seul endroit où Anouilh se permet du lyrisme et une certaine inclination pour le personnage de Bitos-Robespierre, est lié aux souffrances qu'il devait subir étant enfant. Il essayait en tant que fils d'une blanchisseuse en vain d'être l'ami du riche Maxime, lui faisait ses devoirs, portait ses affaires, mais celui-ci le détestait, de fait qu'il manquait de grâce, ce qui a d'ailleurs duré toute sa vie. La pitié pour les pauvres opprimés forme un thème cher au dramaturge Jean Anouilh.

Enfin, le personnage de Robespierre permet à l'auteur d'exprimer ses opinions critiques envers toutes les sociétés en général et leurs pratiques politiques. Il appelle la république une démocratie du verbe et s'en prend à l'activité principale des révolutionnaires qui était leurs discours. « La moindre interruption était fatale. Quand on vous coupait la parole, on vous coupait également la tête [...] Comment croyez-vous qu'on ait eu Robespierre ? En faisant assez de bruit pour couvrir sa 
voix. » (ANOUILH, $1958: 390$ ) L'auteur ne crée pas une pièce contre la Révolution française, ce qu'on lui a reproché, mais contre la politique et l'excès des idéologies. «En France [...] on tue, soit, mais on y met des formes. L'ordre, toujours l'ordre [...] La poigne! C'est le seul secret. Et pas besoin du gant de velours. La main de fer suffit... Mais attention! Pour le bien de peuple [...] L'arbitraire des rois est un crime! L'arbitraire des peuples ou de ceux qui le représentent est sacré. » (ANOUILH, 1958: 488, 457) Dans l'interprétation du personnage de Robespierre se reflète le thème anouilhien omniprésent (très travaillé par exemple déjà dans son Antigone), celui de la culpabilité commune et de la responsabilité pour des actes commis par l'individu au nom d'une idée régnante. Ainsi Bitos n'est pas un assassin mais un représentant du pouvoir qui ne fait que son devoir. D'après Anouilh, la vertu était dans l'histoire de la France beaucoup plus dangereuse que les crimes et « [...] ceux qui parlent trop souvent de l'humanité ont une curieuse tendance à décimer les hommes. » (ANOUILH, 1958 : 412).

On peut constater que l'image anouilhienne de Robespierre, même si elle est beaucoup plus négative, est plus vraisemblable et touchante, et elle peut provoquer une certaine compassion pour un héros négatif, abandonné et malheureux. Il est évident qu'Anouilh, le maitre de l'ambiguïté, utilisant une approche tout à fait opposée à celle de Romain Rolland, a provoqué par son personnage plus de sentiment et pour lui plus d'inclination que Rolland par son exaltation invraisemblable, même si décrite en toute sincérité par l'auteur. Certes, l'interprétation anouilhienne est plus récente et donc plus moderne et proche du lecteur d'aujourd'hui. Jean Anouilh parodie la Révolution, il prend à Robespierre sa grandeur, il ne s'agit même pas du mythe du monstre, il l'affaiblit, le démythise et son personnage ne provoque plus de grandes passions comme l'admiration ou la haine mais tout simplement la pitié. Tandis que l'intention de Rolland était évidente, celle de célébrer le héros national Robespierre, conformément à son cycle du théâtre de la révolution, et de le montrer aux générations futures, Jean Anouilh s'est intéressé à Robespierre indirectement, à travers la psychologie du personnage parallèle de Bitos, le double du caractère de Robespierre.

Le personnage de Robespierre de Romain Rolland devient ainsi plus schématique et moins vivant, chez Anouilh il se montre plus complexe, ses faiblesses humaines sont plus découvertes. Si l'interprétation rollandienne offre une image de Robespierre qui est assez claire, nette et touche à l'idéalisation et à la mythisation du héros, la vision anouilhienne mène à travers cette double face du mythe, l'image du monstre tyrannique, à une démythisation de ce personnage.

Les deux auteurs essaient de pénétrer dans les origines du caractère du personnage, de découvrir ses côtés intimes, son enfance qui détermine son comportement. Si Romain Rolland manifeste toujours une certaine compassion pour son héros, Anouilh, beaucoup plus sévère avec lui, montre ses côtés négatifs qui ont déformé son caractère. Il ne s'attendrit que devant sa pauvreté et l'humiliation qu'il a dû subir pendant son enfance et donne ainsi une interprétation plutôt ambiguë du héros, le monstre Robespierre qui provoque la pitié. Romain Rolland introduit aussi les scènes de l'enfance de Robespierre. Les deux auteurs situent ce moment lyrique avant la mort du personnage, et les deux choisissent un intermezzo dramatique encadré par le changement original de la perspective - 64 - 
dramatique dans le jeu de l'ombre où Anouilh fait prononcer à un acteur adulte les paroles d'un acteur enfant et le héros de Rolland assiste, comme en rêve, à des scènes les plus signifiantes de son enfance.

Jean Anouilh fait une analyse psychologique plus profonde de l'âme de ce héros et à la différence du monde rollandien presque totalement masculin, Anouilh, dans son œuvre, donne aussi une place importante aux femmes qui entourent son héros et essaie de pénétrer dans les relations entre Robespierre et ses compagnons ainsi que de découvrir les motivations profondes de leurs actions. La vision anouilhienne se fait pessimiste et parfois même cynique. Romain Rolland, à son tour, excelle dans la description des scènes du peuple et de la manipulation de la foule. Il décrit à la perfection l'enthousiasme sans limites de gens vivant dans une époque transcendante. Les deux auteurs, chacun à leur manière, ont créé un grand théâtre historique et à travers le personnage de Robespierre ont exprimé leurs vérités personnelles et apporté leur vision originale et sincère du monde.

\section{BIBLIOGRAPHIE}

Anouilh Jean (1958), Pauvre Bitos ou Le Dîner de têtes, Pièces grinçantes, Paris, La Table ronde.

BRUNEL Pierre (1988), Dictionnaire des mythes littéraires, Monaco, Editions du Rocher.

DuCHATELET Bernard (2002), Romain Rolland tel qu'en lui-même, Paris, Albin Michel.

GINESTIER Paul (1969), Jean Anouilh, Paris, Editions Seghers.

MERCIER Christophe (1995), Pour saluer Jean Anouilh, Paris, Bartillat.

MAUROIS André (1958), Histoire de la France, Paris, Albin Michel.

MALACHY Thérèse (1978), Jean Anouilh, les Problèmes de l'existence dans un théâtre de marionnettes, Paris, Nizet.

NovÁK Otakar (1958), Pět studií o Romainu Rollandovi [Cinq études sur Romain Rolland], Po stopách realistických tradic francouzského písemnictví, Brno, SPN.

ROLLAND Romain (1939), Robespierre, Paris, Albin Michel.

ROLLAND Romain (1903), Le théâtre du peuple, Paris, Cahiers de la Quinzaine.

Rolland Romain (1926), Théâtre de la Révolution. Le 14 Juillet. Danton. Les Loups, Paris, Librairie Ollendorff. 


\begin{abstract}
Historians perceive the real personality of Maxmilian de Robespierre in several considerably different ways. This perception is reflected also in his literary depiction. It is possible to speak about the personality of Robespierre as a literary myth which has two faces. On one hand, it is a myth of a revolutionary - saviour sacrificing himself for the well-being of society. On the other hand, it is a heartless monster hankering after human flesh. Our paper deals with the conception of the personality of Robespierre in two ideologically contradictory authors - Romain Rolland and Jean Anouilh in their works Le 14 juillet, Danton, Robespierre and Pauvre Bitos ou Le Dîner de têtes. Whereas Rolland's conception of the personality of Robespierre is ideologically based, Jean Anouilh pulls down the myth of Robespierre, offering an equivocal interpretation of this personality. On one hand, Anouilh condemns the violation committed on the individual; on the other hand, he speaks about him with a highly human understanding.
\end{abstract}

